\title{
ESOPHAGOGASTRODUODENOSCOPY: A BINDING TOOL FOR FOLLOW-UP OF ESOPHAGEAL ATRESIA PATIENTS
}

\author{
Radu Spătaru $^{1}$, Dan Iozsa ${ }^{1}$, Lorena Vatra ${ }^{1}$, Monica Ivanov ${ }^{1}$, Sebastian Ionescu ${ }^{1}$
}

\section{Abstract}

Follow-up of pediatric esophageal atresia (EA) patients in Romania is relatively difficult due to insufficient access to proper tools and special trained health professionals. We address this paper to increase the consciousness in the both medical and patient community over the relevance of providing close upper endoscopic evaluation in EA children so the best results in long-term complications identification and treatment may be obtained. Possible critical complications in infancy, longstanding to adult life can be averted by prevention and a wellstandardized evaluation plan. A series of 4 cases operated and currently followed up for EA in our clinic were selected. Their history, radiologic and endoscopic evaluation are pointed out willing to extract conclusive observations over the esophagoduodenoscopy position in these patients. The most frequent condition following EA surgery is gastroesophageal reflux disease (GERD). Treatment of GERD should be tailored accordingly to its extent, nature and long-term evolution in order to avoid unnecessary surgery. Left unidentified, incompletely evaluated and subsequently treated this may lead to serious complications like epitheal metaplasia or peptic esophageal strictures in which using flexible esophagoscopy and a good focus over the the esophageal configuration and dilatations should be the prime option of their treatment. Upper endoscopy is required to be regularly considered in a constant standardized follow-up of EA patients, depending on their evolution, from the early ages to adulthood, thus a good quality of life is ensured and - in latter life - a better transition to adult medical or surgical care is done.

Keywords: Esophageal atresia complications, endoscopy, gastroesophageal reflux, esophageal stricture

\section{Introduction}

"A monstrous birth in Plymouth" had been described in 1670 by Durston who was mentioning in his necropsy report "the oesophagus from the mouth of the right head descended no lower than a little above half an inch off the midriff, and there it ended" in a female thoracopagus twin [1]. 199 years after, Holmes suggests that an attempt of esophageal atresia (EA) repair may be made. In 1939 Ladd and Leven have reported the first operated esophageal atresia survivors triggering the ascension of EA surgical treatment outcomes together with the progresses made in neonatal intensive care. Therefore, in 1962 The Lancet journal has published Waterston's paper studying 218 EA infants and considering birthweight, pneumonia and existence of associated congenital anomalies prognostic factors for survival [3] and later, in 1994, Spitz reconsiders these specifying only birthweight and the existence congenital heart anomaly as patients at risk [4]. Later on, in 2009, a team Okamoto T. revisits Spitz's classification and reconsiders stratification of EA patients' survival depending only on birthweight and major cardiac anomalies [5].

Accompanying the progress made in EA patients' survivability, Puri's observations over the possibility of delayed primary anastomosis following spontaneous growth of esophageal segments in EA in 1981 [6] and - in 1992 the positive outcomes after applying this principle [7] constitute a huge twist in EA surgical treatment, esophageal substitution becoming subsidiary and a new vision over the follow-up and late complications of these patients has quickly emerged. Most pediatric surgeons consider today that native esophagus preservation provides the most physiological results in matter of reconstruction. In consequence, the challenge of the esophageal gap associated to the surgeon's ambition toward primary esophageal anastomosis - is also a harbinger for gastroesophageal junction displacement into the thorax, leading practically to cardia incompetence, long-term GER and strictures in most of the cases [8]. Nevertheless, surgical repair of esophagus itself disrupts the esophageal motility leading to dysmotility who to its turn leads to a greater chance of developing gastroesophageal reflux (GER) [9].

\section{Material and method}

Personal observations disclose a difficult follow-up and proper diagnosis of EA complications in Romania. This may be associated to the low parents' compliance, their poor health literacy, but also by a burdensome access to specialized health care (pediatric gastrointestinal endoscopy) because of defective medical infrastructure, lack of high-performance devices and specialized pediatric health professionals. Throughout this paper we wish to raise the awareness among both pediatric professionals and patient population over the fundamental role played by upper endoscopy as a follow-up, diagnosis and treatment tool in long term esophageal atresia management.

${ }^{1}$ Pediatric Surgery Clinic of the Emergency Clnical Hospital for Children „Marie S. Curie”, Bucharest, Romania E-mail: dan.iozsa@yahoo.com,nsionescu@yahoo.com, lorena.vatra@rodelta.ro,mqmivanov@yahoo.com, radu_spataru@yahoo.com 
In this manner, a series of 4 relevant cases in our clinic's experience in evaluation of long-term EA complications. We bring into discussion their history, radiologic or endoscopic features, emphasizing on the contribution upper endoscopy has made in these willing to point out its value.

\section{Results \\ Case 1}

A 10 years old male patient is admitted for his first endoscopic evaluation 8 years prior transverse colon esophageal replacement procedure for EA. He doesn't report any feeding or swallowing difficulties or history of GERD symptoms. Last esophagogram obtained doesn't objectify episodes of GER. Upper endoscopy is performed noticing persistent biliary reflux at the distal level of the colonic graft. Even more, intestinal metaplasia changes (glandular layout accentuation) are noticed at the level of distal esophageal-colic anastomosis (Fig. 1). Biopsy specimens are obtained and the diagnosis is histopathologically confirmed.

\section{Case 2}

A 4 years old girls known with postoperative GER after delayed primary anastomosis for EA is admitted for her second esophageal endoscopic evaluation. Diagnosis of grade B (Los Angeles) erosive esophagitis in a previous evaluation 6 months before current evaluation. Mucosal tears healing could be evaluated and the healing process confirms the efficacy of proton pump inhibitor dosage adopted so far (Fig. 2).

Case 3

An exceptionally rare case of a 4 years old female patient with esophageal atresia with distal tracheoesophageal fistula (TEF) associating congenital microgastria is managed in our pediatric surgery clinic. Ligation of the fistula and gastrostomy were done at birth and delayed primary esophageal anastomosis was performed after. High persistent GER in association with peptic anastomotic stricture quickly developed and re-do anastomosis had to be done. The gastric malformation was corrected at 1 year old considering a Hunt-Lawrence gastric augmentation procedure and replacement of the gastrostomy. During the follow-up, esophageal anastomosis stricture recurs, but patient's age and weight allows establishing a safe Savary esophageal dilatation treatment plan. Long term high dose PPIs is adopted complementary because of persistence of high GERD. Routine barium meal was done after apparently a favorable outcome of esophageal dilatations in order "to avoid unnecessary anesthesia" and opportunity of gastric tube removal showing a satisfying aspect of the esophageal anastomosis. However, the patient is shortly readmitted after the radiologic evaluation with dysphagia, upper esophagus food impactation and a punctiform esophageal stricture (Fig. 3). After dilatation of the anastomotic stricture a second stricture could be visualized endoscopically presenting a voluminous hiatal hernial below with passive gastric content reflux (Fig. 4). The patient is currently under endoscopic surveillance and dilatations following spontaneous evolution of GERD.

\section{Case 4}

An 11 years old male is admitted in the pediatric surgery clinic for dysphagia. History of the patient relates: primary esophageal anastomosis for EA with distal TEF and re-do procedure for esophageal anastomosis stricture in his first year of life which quickly recurred and required gastrostomy, further on being treated by Foker tractions and Nissen fundoplication in a pediatric surgery clinic abroad. When the second esophageal anastomosis is attempted dehiscence occurs and the patients' parents decide to come to our clinic where a third re-do esophageal anastomosis is complicated shortly after by stricture formation. The parents decide to follow dilatation treatment through rigid esophagoscopy in the ENT department with a satisfactory outcome on barium swallow evaluation imagery and in matter of quality of life - "the kid eats well, sometimes he chokes shortly, but he handles it". The patients present to pediatric surgery department and flexible endoscopy is done for the first time: eccentric esophageal stricture with giant pre- anastomosis stricture diverticula formation is noted (maybe due to blind rigid esophagoscopic dilatation), high quantity of partially digested food residue in the diverticula (Fig. 5). After Savary dilatation is done the endoscope can be advanced through the anastomosis and Los Angeles grade D erosive reflux esophagitis is demonstrated (Fig. 6). Re-do anastomosis was done considering the refractory diverticulum excision and dilatation program and high dose GERD treatment plan is currently considered.

\section{Discussions}

More than $90 \%$ of the EA patients survive today, their only poor prognostic being dictated solely by severe cardiac malformations or low birth weight. Nearly half of the patients present GER up to 10 years after primary esophageal reconstruction and $25 \%$ to $75 \%$ of the adults and children with repaired EA associate chronic GER symptoms. $53 \%$ of long-term followed-up EA patients present erosive esophagitis and they have a 4-fold higher risk than normal population of developing Barrett's esophagus [10]. Moreover, Sri Rapan reports in 2007 an incidence of the strictures of $76 \%$ in delayed primary esophageal anastomosis of which $23 \%$ needed resection [8]. Esophageal adenocarcinoma has also been scarcely reported in the literature following EA repair [10].

Gastroesophageal reflux disease (GERD) clinical picture is often subtle in children and barium contrast study in its follow-up and diagnosis is not recommended due to its low sensibility and specificity. GERD may be hidden under less specific symptoms like dental erosions, chronic cough, raspy voice, recurrent pneumonia or otitis, apnea, asthma, chronic anemia, Sandifer Syndrome or failure to thrive [11]. Upper endoscopy with or without tissue biopsy may detect and follow-up GERD development throughout macroscopic evaluation to minor microscopic architecture modifications, some of them of substantial importance like intestinal metaplasia or Barrett's esophagus. 


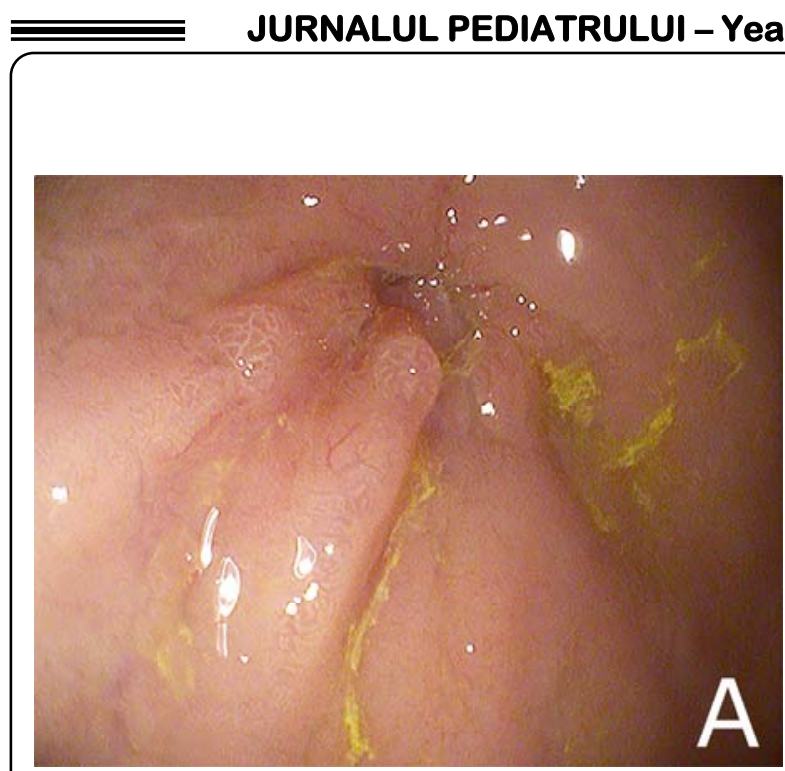

Fig. 1. Intestinal metaplasia change

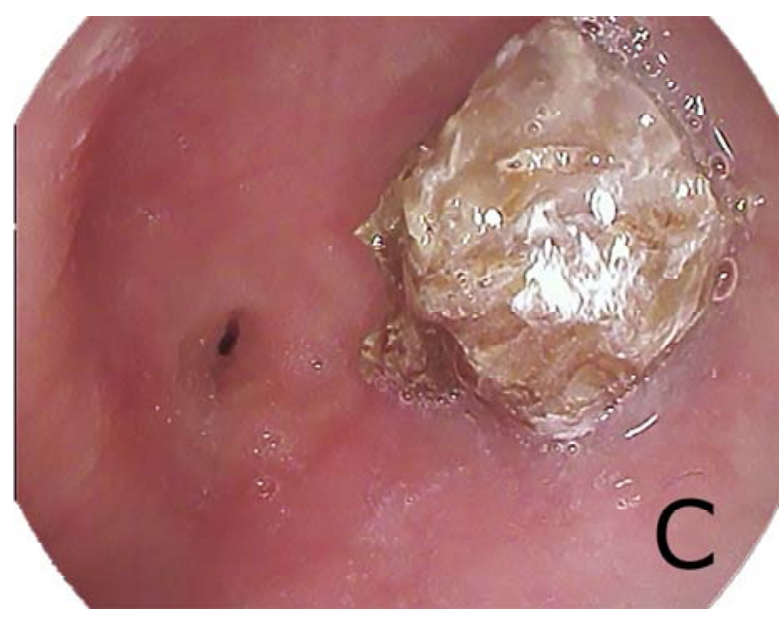

Fig. 3. Upper esophagus food impactation and a punctiform esophageal stricture

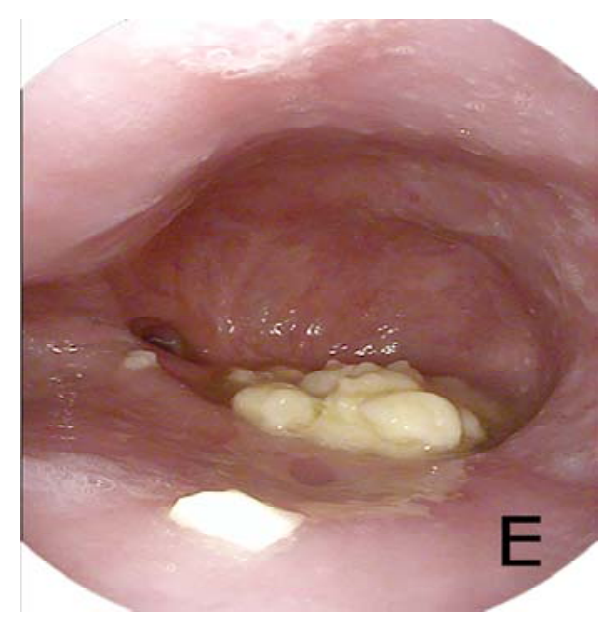

Fig. 5. Excentric esophageal stricture with giant pre- anastomosis stricture diverticula

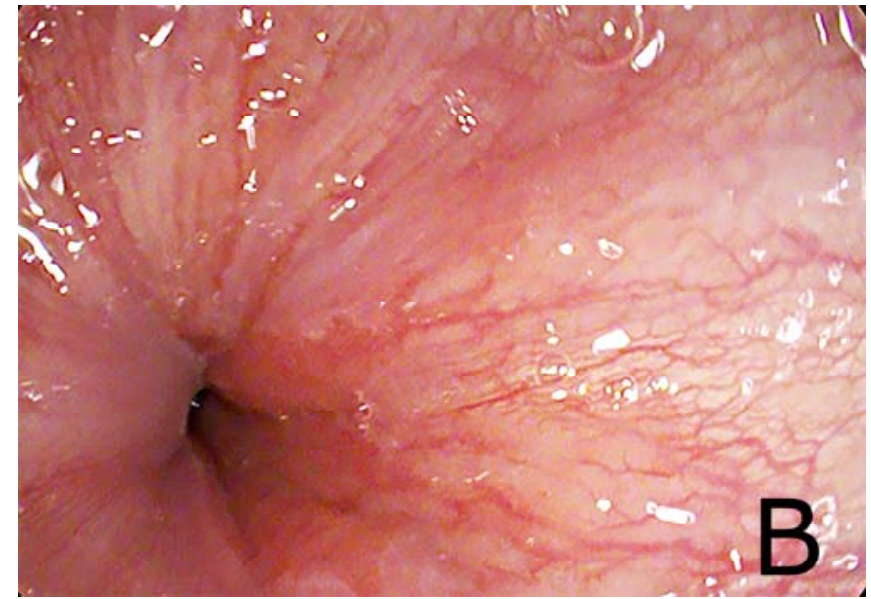

Fig. 2. Mucosal tears healing

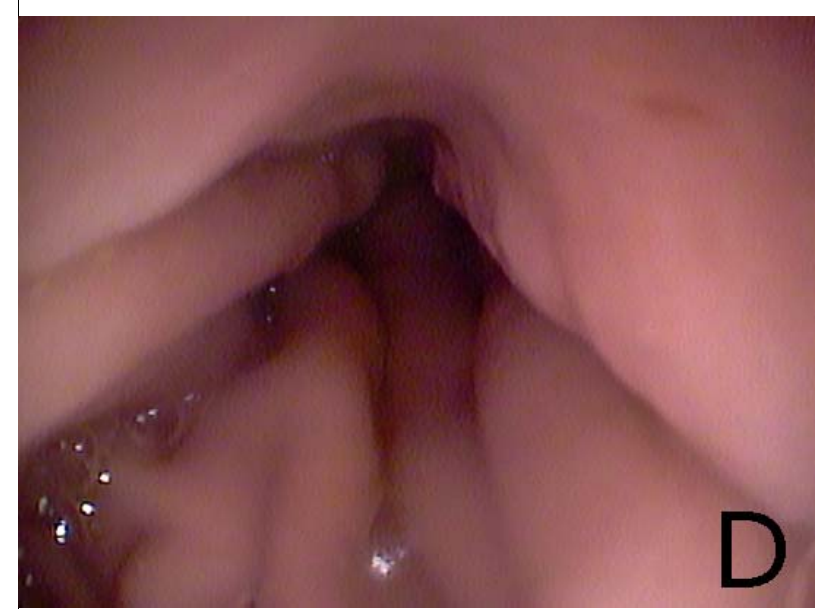

Fig. 4. Voluminous hiatal hernial below with passive gastric content reflux

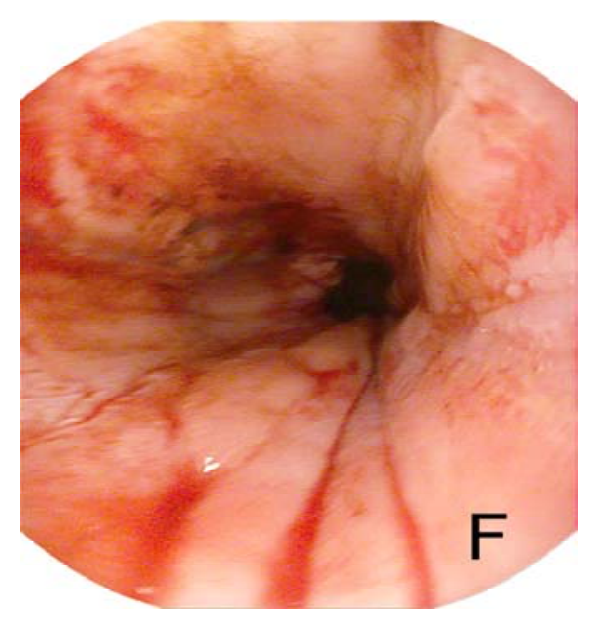

Fig. 6. Los Angeles grade D erosive reflux esophagitis 
Postoperative endoscopic assessment of EA patients is recommended even if the symptoms are regardless [12]. By resemblance, later in adult life, even if the patients report a very good quality of life or absence of GERD symptoms, this is not an indicator for a healthy esophagus and asymptomatic patients are often reported presenting severe forms of GER. There's also a proof of a poor relationship between subjective GER symptoms and pathological GER identified at pH-metry [13].

Anastomotic stricture formation process in EA may be intensified by the presence of gastric juice. GER is precipitated by displacement of the esophagogastric junction while mobilization the distal esophagus and by esophageal dysmotility related to EA. [9, 14]. Contact with acid fluid seems to intensify regeneration process from which a higher chance to develop an anastomotic stricture. Moreover, chronic exposure to acid secretions may induce de novo peptic strictures. Therefore, PPI treatment is routinely recommended after an EA anastomosis, even if the patient doesn't present any signs of GER. A considerable amount of EA patients (8-15\%) still deal with anastomotic strictures in adulthood. When you deal with an anastomotic stricture esophageal dilatation using flexible endoscopy should be the first line of treatment [14]. Dilatations are most often required the first two years after primary repair [15]. Bougie or balloon dilators can be used, but currently there's no strong evidence if one or other is more efficient and, therefore is all up to the endoscopist's experience and comfort. In case of recurrent or refractory anastomotic strictures, several adjuvant methods are known: systemic or intralesional steroid therapy, intravenous mitomycin $\mathrm{C}$, endoscopic electrocautery insicional treatment or esophageal stenting. Surgical management like stricture resection or esophageal replacement should be reserved for exceptional refractory cases [14].

EA patients represent a special subgroup when it comes to indication and type of anti-reflux surgery. Wrap failure is more common in these patients, even if initially there is a prompt symptoms' relief. There are not enough scientific proofs to delineate the efficacy of a complete wrap versus partial wraps, but the surgeon should always keep in mind the dysphagia, retching or gas-bloating a Nissen fundoplication may lead to and balance this with the infant's natural evolution of GERD, esophageal dysmotility, coexistence of strictures or delayed gastric emptying. Accordingly, long term PPI with a close followup of its efficacy should always be considered before any indication of anti-reflux procedure [16].

In addition, evaluating esophageal strictures through direct flexible endoscopy a full image over the whole esophagus anatomy, the stenosis elasticity, localization, associated anomalies (like large upper pouches, hiatal hernia, esophageal diverticula) while U-turn evaluation of the gastric fornix may bring relevant information about the looseness or efficacy of the anti-reflux procedure.

\section{Conclusions}

Endoscopy should be considered a gold standard tool for long-term follow-up and treatment in EA children and it should be integrated systematically in EA management protocols.

A more meticulous detailed long-term evaluation of EA leads to a better prevention of imminent complications, consequently a better quality of life and, nevertheless, a soft and clean transition of the patient to adult healthcare professionals.

\section{References}

1. Myers NA. (1986) "The History of Oesophageal Atresia and Tracheo-Oesophageal Fistula - 1670-1984", in Rickham P.P. (eds) Historical Aspects of Pediatric Surgery. Progress in Pediatric Surgery, vol 20, Springer, Berlin, Heidelberg

2. Spitz L. Esophageal atresia. Lessons I have learned in a 40-year experience. J Ped Surg 2006. 41: 1635-1640.

3. Waterston DJ, Bonham Carter RE, Aberdeen E. Oesophageal atresia: trachea-oesophageal fistula. A study of survival in 218 infants. Lancet. 1962; 1(7234):819-22.

4. Spitz L, Kiely EM, Morecroft JA, Drake DP. Oesophageal atresia: at-risk groups for the 1990s. J Ped Surg. 1994; 29(6): 723-5.

5. Okamoto T, Takamizawa S, Arai H, Bitoh Y, Nakao M, Yokoi A, Nishijima E. Esophageal atresia: prognostic classification revisited. Surgery. 2009; 145(6): 675-81.

6. Puri P, O’Donnell B, Guiney EJ. Delayed primary anastomosis following spontaneous growth of

esophageal segments in esophageal atresia. J Ped Surg. 1981; 16(2): 180-3.

7. Puri P, Ninan GK, Blake NS, Fitzgerald RJ, Guiney EJ, O'Donnell B. Delayed primary anastomosis for esophageal atresia: 18 months' to 11 years' follow-up. J Ped Surg. 1992; 27(8): 1127-1130.

8. Sri Paran T, Decaluwe D, Corbally M, Puri P. Long term results of delayed primary anastomosis for pure oesophageal atresia: a 27-year follow up. Pediatr Surg Int. 2007; 23: 647-651.

9. Sistonen $\mathrm{S}$, Koivusalo A, Nieminen $\mathrm{U}$, Lindahal $\mathrm{H}$, Lohi J, Kero M, Paivi A, Karkkainen PA, Marti AF, Sarna S, Rintala RJ, Pakarinen MP. Esophageal morbidity and function in adults with repaired esophageal atresia with tracheoesophageal fistula. A population-based long-term follow-up. Ann Surg. 2010; 251: 1167-1173.

10. Rintala RJ, Sistonen S, Pakarinen MP. Outcome of esophageal atresia beyond childhood. Semin Pediatr Surg. 2009; 18(1): 50-56. 
11. Rosen R, Vanderplas Y, Singendonk M. Pediatric Gastroesophageal Reflux Clinical Practice Guidelines: Joint Recommendations of the North. American Society for Pediatric Gastroenterology, Hepatology, and Nutrition and the European Society for Pediatric Gastroenterology, Hepatology, and Nutrition. 2018; 66(3): 516-554.

12. Schalamon J, Lindahl H, Saarikoski, Rintala RJ. Endoscopic follow-up in esophageal atresia - for how long is it necessary? J Ped Surg. 2003; 38(5): 702-4.

13. Maynard S, Bouin S. Follow-up of adult patients with repaired esophageal atresia: how, when and for how long? Dis Esophagus. 2013; 26: 422-4.

14. Tambucci R, Angelino G, De Angelis P, Torroni F, Caldaro T, Balassone V, Contini AC, Romeo E, Reaf F, Faraci S, Di Abrioli GF, Dall'Oglio L. Anastomotic strictures after esophageal atresia repair: incidence, investigations and management, including treatment of refractory and recurrent strictures. Front Pediatr. 2017; 5(120):1-14.

15. Stenstrom $P$, Anderberg M, Borjesson A, Arnbjornsson E. Dilations of anastomotic strictures over time after repair of esophageal atresia. Pediatr Surg Int. 2017; 33:191-5.

16. Rintala RJ. Fundoplication in patients with esophageal atresia: patient selection, indication and outcomes. Front Pediatr. 2017; 5(109):1-5.

Correspondence to:

Dan Iozsa

Pediatric Surgery Clinic

Emergency Clnical Hospital for Children „Marie S. Curie”, 20 C-tin Brâncoveanu Blvd., Bucharest, Romania

E-mail: dan.iozsa@yahoo.com 